\title{
Enhanced low density lipoprotein-cholesterol lowering efforts by consuming plant stanols as part of a low saturated fat diet
}

\author{
C. Shortt ${ }^{1}$, K. Musa-Veloso ${ }^{2}$ and T. Poon ${ }^{2}$ \\ ${ }^{1}$ McNeil Nutritionals Ltd, London, W1A $5 X Y$, UK and ${ }^{2}$ Intertek Cantox, Mississauga, Canada
}

It is well recognised that decreased saturated fat intake independently reduces low-density lipoprotein cholesterol (LDL-C) levels, irrespective of the type of macronutrient that is used to replace dietary saturated fat. In addition, it is acknowledged that daily intakes of 1.5 to $2.4 \mathrm{~g}$ of plant stanols/plant sterols lead to reductions in LDL-C of 7 to $10 \%$ within 2 to 3 weeks. Individuals who wish to maintain healthy cholesterol levels are increasingly interested in the combined effects of dietary approaches to blood lipid level reduction.

A systematic evidence-based review was conducted to determine the benefits of consuming $2 \mathrm{~g} /$ day of plant stanols as part of a diet low in saturated fat compared to the benefits of consuming a low saturated fat diet alone. Using the electronic search tool Dialog ${ }^{\circledR}$, a comprehensive literature search of 8 databases was undertaken to retrieve relevant human studies wherein the combined efficacy of consuming a diet low in saturated fat and $2 \mathrm{~g}$ /day of plant stanols (as plant stanol ester) on LDL-C levels was assessed. Only studies that met all of the pre-defined inclusion criteria and none of the pre-defined exclusion criteria were included in the assessment. A diet low in saturated fat was defined as a dietary saturated fat intake of $\leq 10 \%$ of the total daily energy intake.

Of the 133 full length publications retrieved, a total of 5 publications (representing 6 strata, all randomised, placebo-controlled trials) were found to be eligible for inclusion in the scientific assessment. Amongst the 6 strata, the target intakes of plant stanols ranged from 1.8 to $2.3 \mathrm{~g} /$ day, and saturated fat intakes were $\leq 10 \%$ of total energy. A 2-fold greater reduction in LDL-C (range 1.6- to 3.4-fold) was observed when plant stanols (delivered as plant stanol ester) were consumed as part of a diet low in saturated fat than when a diet low in saturated fat was consumed alone. The percentage relative LDL-C changes after consuming plant stanols as part of a low saturated fat diet ranged from -7.9 to $-23.6 \%$ and after consuming a low saturated fat diet alone ranged from -3.9 to $-12.0 \%{ }^{(1-5)}$. Of the 5 strata where differences between the groups were recorded, statistical significance was reached in 4 of the groups $(P<0.05)$.

Although the LDL-C lowering mechanism of plant stanol ester remains to be fully elucidated, its role in the suppression of intestinal cholesterol absorption complements the mechanism of LDL-C lowering resulting from a reduced intake of dietary saturated fat. The results show that when consumers combine efficacious dietary approaches to lower LDL-C the effect is enhanced compared to that obtained from a single dietary approach.

1. Andersson A, Karlstrom B, Mohsen R et al. (1999) Eur Heart J 1, S80-S90.

2. Athyros V, Kakafika A, Papageorgiou A et al. (2011) Nutr Metab Cardiovasc Dis 20, $213-221$.

3. Halikainen M, Uusitupa M (1999) Am J Clin. Nutr 69, 403-410.

4. Halikainen M, Sarkkinen E, Gylling H et al. (2000) Eur J Clin Nutr 54, 715-725.

5. Jones R, Raeini-Sarjaz M, Ntanios F et al. (2000) J Lipid Res 41, 697-705. 